\title{
Erratum to: Comparison of Pulmonary Involvement Between Patients Expressing Anti-PL-7 and Anti-Jo-1 Antibodies
}

\author{
Masaomi Tomonaga $^{1,2} \cdot$ Noriho Sakamoto $^{1} \cdot$ Yuji Ishimatsu $^{1} \cdot$ Tomoyuki Kakugawa $^{1} \cdot$ \\ Tatsuhiko Harada $^{1} \cdot$ Shota Nakashima $^{1}$ - Atsuko Hara ${ }^{1}$ - Shintaro Hara ${ }^{1}$. \\ Yoshihiro Horai $^{3} \cdot$ Atsushi Kawakami $^{3} \cdot$ Hiroshi Mukae $^{4} \cdot$ Shigeru Kohno $^{1}$
}

Published online: 23 July 2016

(C) Springer Science+Business Media New York 2016

\section{Erratum to: Lung (2015) 193:79-83 DOI 10.1007/s00408-014-9665-7}

In the original publication of the article, the coauthor Masaomi Tomonaga was affiliated with "Second Department of Internal Medicine, Nagasaki University School of Medicine, 1-7-1 Sakamoto, Nagasaki 852-8501, Japan”. However, the author would like to add the following affiliation as well.
The new affiliation is: Department of Infectious Diseases, Unit of Molecular Microbiology and Immunology, Nagasaki University Graduate School of Biomedical Sciences, Nagasaki, Japan.

This erratum is issued to add Dr. Tomonaga's new affiliation.

The online version of the original article can be found under doi:10.1007/s00408-014-9665-7.

Yuji Ishimatsu

yuji-i@nagasaki-u.ac.jp

1 Second Department of Internal Medicine, Nagasaki

University School of Medicine, 1-7-1 Sakamoto,

Nagasaki 852-8501, Japan

2 Department of Infectious Diseases, Unit of Molecular Microbiology and Immunology, Nagasaki University Graduate School of Biomedical Sciences, Nagasaki, Japan

3 Unit of Translational Medicine, Department of Immunology and Rheumatology, Nagasaki University Graduate School of Biomedical Sciences, Nagasaki, Japan

4 Department of Respiratory Medicine, University of Occupational and Environmental Health, Kitakyushu, Japan 